\title{
Does the distribution of the weekly training load account for the match results of elite professional soccer players?
}

The corrections made in this section will be reviewed and approved by a journal production editor.

Rafael Oliveira ${ }^{\mathrm{a}, \mathrm{c}, \mathrm{d},{ }_{\star}}$ rafaeloliveira@esdrm.ipsantarem.pt, João P. Brito ${ }^{\mathrm{a}, \mathrm{d}}$, Nuno Loureiro ${ }^{\mathrm{a}, \mathrm{d}}$, Vitor Padinha ${ }^{\mathrm{a}}$, Bruno Ferreira ${ }^{\mathrm{a}}$, Bruno Mendes ${ }^{\mathrm{b}}$

aESDRM-IPS - Sports Science School of Rio Maior - Polytechnic Institute of Santarém, Av. Dr. Mário Soares, Rio Maior 2040-413, Portugal

${ }^{\mathbf{b}}$ Falculty of Human Kinetics, University of Lisboa, Lisboa, Portugal

${ }^{c}$ CIDESD - Research Centre in Sport Sciences, Health Sciences and Human Development, Quinta de Prados, Edifício Ciências de Desporto, Vila Real 5001-801, Portugal

${ }^{\text {d} C I E Q V ~-~ L i f e ~ Q u a l i t y ~ R e s e a r c h ~ C e n t r e, ~ C o m p l e x o ~ A n d a l u z, ~ A p a r t a d o ~ 279, ~ S a n t a r e ́ m ~ 2001-904, ~ P o r t u g a l ~}$

*Corresponding author at: Sports Science School of Rio Maior - Polytechnic Institute of Santarém, Av. Dr. Mário Soares, Rio Maior 2040-413, Portugal.

\begin{abstract}
Purpose.: : The aim of the study was to compare training load (TL) of the days preceding a win, draw or defeat in a sample of elite professional soccer players across the in-season 2015/16.

Methods.: : Twenty elite soccer players participated in this study. Total distance covered, highspeed running distance (HSRD), average speed, session rate of perceived exertion (s-RPE) and Hooper index scores (HI) were collected. Data from 24 weeks with one match were analysed through the match-day (MD-5, 4, 3, 2, 1) and MD+1.

Results. $:$ : The main finding emerges in MD-1, where a longer training duration preceding draws $\left(95.1 \_ \pm 1.5 \mathrm{~min}\right)>$ defeats $\left(91.5 \_ \pm 1.6 \mathrm{~min}\right)>$ wins $\left(84.7 \_ \pm 0.5 \mathrm{~min}\right)$ was found, while total distance and average speed were higher in wins $\left(3628.6 \_ \pm 57.2 \mathrm{~m}\right)>\operatorname{draws}\left(3391.3 \_ \pm_{-} 153.3 \mathrm{~m}\right)>$ defeats $\left(3236.1 \_ \pm \_113.7 \mathrm{~m}\right)$ and draws $\left(130.7 \_ \pm \_17.6 \mathrm{~m} / \mathrm{min}\right)>$ wins $\left(86.0 \_ \pm \_6.9 \mathrm{~m} / \mathrm{min}\right)>$ defeats $\left(54.8 \pm_{-}{ }_{-} 7.1 \mathrm{~m} / \mathrm{min}\right)$, respectively. HSRD was higher in draws $\left(42.8 \pm_{-} \pm_{-} 0.6 \mathrm{~m}\right)>$ wins $\left(36.1 \pm_{-} \pm_{-} 1.7 \mathrm{~m}\right)$ $>$ defeats $\left(35.8 \pm_{-} 1.7 \mathrm{~m}\right)$. In MD+1, there were differences in HI between wins vs draws $(p<0.01)$.
\end{abstract}

Conclusions.: : The results are drawn from one team that participated in UEFA Champions League. It was observed that different TL applied in training sessions can influence match result. Our 
findings can be considered in future soccer planning and periodization to win matches. This study emphasizes the use of HI especially in the day following the match.

Keywords: Soccer training; Internal load; External load; Training load; Match result-

\section{Introduction}

Elite soccer teams play one to three matches per week due to the demanding match schedule [1-4]. Consequently, accumulated fatigue can occur and impair match outcome [5]. Therefore, it is very important to monitor internal and external training load (TL) to prevent injury (29\%), to promote effectiveness of the training programme (27\%), to maintain performance (22\%), to prevent overtraining (22\%) [6] and to model and possibly predict the effect of training load on performance [7].

Monitoring the wellness of athletes allows us to know the details of fatigue, stress, delayed onset muscle soreness (DOMS), need for recovery and sleep perception [8]. The Hooper index [9] assesses these variables (fatigue, stress, DOMS and quality of sleep) which are associated with biochemical (physical and physiological) and biomechanical stress responses, recognized as internal training load [8]. Another complementary variable used to assess internal TL is session-RPE (s-RPE), proven to be valid and reliable in correlation with the heart frequency training zones [10]. However, it is not sensitive to the subjective perceptions of fatigue, DOMS or stress [11], which makes the Hooper index an easier and more complete method of accessing the perceptions mentioned above.

Apart from the afore mentioned studies, the relationship between the use of the Hooper index and s-RPE is limited. Haddad et al. [11] observed no association between the Hooper index and RPE and, recently, Clemente et al. [12] found that the relationship between s-RPE and HI revealed significant and negative small-to-moderate correlations in the weeks with two matches but not in the weeks with only one match. They also reported that HI and s-RPE differed significantly from position to position. However, further research is needed on this issue to validate and generalize the findings regarding an in-season period.

Monitoring the external TL is also critical to assess the physical work performed during the training session or match [8]. We can find several studies in the literature that have been carried out during the in-season with different periods. For example, short training microcycles of $1-2-3$ weeks $[1,8,12,13]$; mesocycles consisting of 4-10 weeks [14,15]; and a 10-month period [5]. Only a few studies [12,16] have attempted to quantify TL with respect to changes between mesocycles and microcycles (both overall and between players' positions) across a full competitive season. Only recently, several studies have described the in-season training periodization practices of elite soccer teams in more detail, including a comparison of training days within weekly microcycles $[1,16,17]$. Nevertheless, there is also currently limited information relating to TL in teams that played in European competitions because the studies do not contemplate the use of internal and external TL variables at the same time.

To the best of our knowledge, only one study has tried to understand if there is any difference between the results of a soccer match (win, draw and defeat) for the internal training load of elite professional players [18] and one regarding internal and external TL combined [4], but none with relation to the match result situation, simultaneously. In order to reach a better sport approach, the match result may influence the external and internal TL applied and vice-versa [19]. We consider it is crucial to understand whether the periodization of the internal and external TL of the microcycles throughout the in-season influences the performance of the players through 
match result (win, draw or defeat). Whilst much of the current research related to soccer has focused primarily on starters and field players, on the demands of regular time of match-play with research related to the most important aspect of the game (match result), it is critical to continue to increase game performance. The match result can impact the training load in the post-game training sessions and vice-versa, either because of the need to recover players or to prepare the team technically and tactically for the next match. The match result and load should be quantified so that coaches and staff can programme appropriate activities to prepare and recover from the demands of match-play. Albeit training loads are the primary driver for cardiometabolic, neuromuscular, and musculoskeletal adaptations, but not all soccer training session is programmed with physical and physiological goals (i.e. technical-tactical sessions).

Therefore, the purpose of this study was to analyse a group of elite professional soccer players that played European competitions during the in-season. Specifically, to quantify the internal and external TL; to compare match day minus for TL preceding a win, draw or defeat; to compare TL between win, draw or defeat results in matches; and to provide some guidelines for periodization in soccer training. We hypothesize that different distribution of training load applied during in-season may influence the next match result.

\section{Materials and methods}

\subsection{Participants}

Twenty elite soccer players of a European team that play in the Champions League with a mean \pm SD age, height and mass of $25.85 \underline{85} \pm-\underline{\underline{1}} 4.55$ years, $183.06 \underline{06} \pm-\underline{\underline{0}} 6.64 \mathrm{~cm}$ and $78.56 \underline{56} \pm-\underline{\underline{ \pm}} 6.64 \mathrm{~kg}$ respectively participated in this study. The sample consisted of four central defenders (CD), four wide defenders (WD), five central midfielders (CM), four wide midfielders (WM) and three strikers (ST). Twelve players included in this investigation were members of their national teams. Inclusion criteria were described in Oliveira et al. [4] study. For further analysis, we add other inclusion criteria to analyse the day following the match (MD+1) by dividing starters and non-starters. It was considered starters if they participated in three consecutive matches for at least 60 min 60 minutes. All participants were familiarised with the training protocols and signed informed consent prior to the investigation. This study was conducted according to the requirements of the Declaration of Helsinki and was approved by the institution's research ethics committee.

\subsection{Design}

TL data were collected over a 39-week period during the 2015-2016 in-season. The team considered for data collection competed in four official competitions across the season, including a European competition, the national league and two more national cups in their own country. For the purposes of the present study, all the sessions carried out as the main team sessions were considered.

Only data from training sessions were considered. Data from rehabilitation or additional training sessions of recuperation were excluded. Total minutes of training session included the warm-up, main phase and slow down phase plus stretching. Due to the inclusion criteria, it was analysed 24 training weeks with one match.

\subsection{Experimental procedures}

Training data collection for this study was carried out at the soccer club's outdoor training pitches. Training data were analysed in relation to the number of days away from the competitive match fixture (i.e. match day minus [MD-]). The data analysed included weeks with one match, where team typically trained five days per week 
(MD-5; MD-4; MD-3; MD-2; MD-1), plus one day after the match $(\mathrm{MD}+1)$. This approach was already in previous work [4]

\subsubsection{External training load - training data}

Each player's physical activity during each training session was monitored using portable global positioning system (GPS) units (Viper pod 2, STATSports, Belfast, UK). This device provides position velocity and distance data at $10 \mathrm{~Hz}$ frequency. Across the upper back between the left and right scapula, each player wore the device inside a custom-made vest supplied by the manufacturer. This position on the player allows the GPS antenna to be exposed for a clear satellite reception. This type of system has previously been shown to provide valid and reliable estimates of instantaneous and constant velocity movements during linear, multidirectional and soccerspecific activities [20]. All devices were activated $30 \mathrm{~min} 30$ minutes before data collection to allow acquisition of satellite signals and synchronise the GPS clock with the satellite's atomic clock [21]. Following each training session, GPS data were downloaded using the respective software package (Viper PSA software, STATSports, Belfast, UK) and were clipped to involve the main team session (i.e. from the beginning of the warm up to the end of the last organised drill). In order to avoid inter-unit error, players wore the same GPS device for each training sessions [22]. The following variables were then selected: total duration of training session, total distance, and high-speed running distance (HSRD, above $19 \mathrm{Km} / \mathrm{h}$ ).

\subsubsection{Internal training load - training data}

Thirty minutes after the end of each training session, players were asked to provide an RPE rating, 0-10 scale [23]. Players were prompted for their RPE individually using a custom-designed application on a portable computer tablet. Each player selected their RPE rating by touching the respective score on the tablet, which was then automatically saved under the player's profile. Individual RPE value was multiplied by the session duration to generate a session-RPE (s-RPE) value [15,24,25].

Approximately $30 \mathrm{~min}$ before each training session, each player was asked to rate the perception of fatigue, stress and DOMS and quality of sleep of the night that preceded the evaluation. The Hooper index scale of 1-7 was used, in which 1 is very, very low and 7 is very, very high (for stress, fatigue and DOMS levels) and 1 is very, very good and 7 is very, very bad (for sleep quality). The Hooper Index is the summation of the four subjective ratings [9].

\subsection{Statistical analysis}

Data were analysed using SPSS version 22.0 (SPSS Inc., Chicago, IL) for Windows statistical software package. Initially descriptive statistics were used to describe and characterize the sample. Shapiro-Wilk and Mauchly's tests were used to assumption normality and sphericity, respectively. ANOVA was used with repeated measures with Bonferroni post hoc, once variables obtained normal distribution (Shapiro-Wilk $>0.05$ ), to compare days away from the competitive match fixture. Also, it was used ANOVA Friedman and MannWhitney tests were used for the variables that not obtained normal distribution to compare different moments and different player positions. Results were significant in the interaction ( $\left.p \leq \leq_{-} 0.05\right)$. The effect-size (ES) statistic was calculated to determine the magnitude of effects by standardizing the coefficients according to the appropriate between-subjects standard deviation and was assessed using the following criteria: $<0.2=$ trivial, 0.2 to $0.6=$ small effect, 0.6 to $1.2=$ moderate effect, 1.2 to $2.0=$ large effect and $>2.0=$ very large [26].

\section{Results}


Descriptive results and comparisons of match day minus for the wins, draws and defeats results of the variables studied, and a comparison of match day minus for Training Load Data between wins, draws and defeats for squad average, are presented in Table 1.

\section{alt-text: Table 1}

\section{Table 1}

Comparison of match day minus for training load data between wins, draws and defeat for squad average $(n=20)$, Mean \pm SD.

\begin{tabular}{|c|c|c|c|c|c|c|}
\hline $\begin{array}{l}\text { MD-5 } \\
(n=24)\end{array}$ & Win & Draw & Defeat & $\begin{array}{l}\text { ES (win vs } \\
\text { draw) }\end{array}$ & $\begin{array}{l}\text { ES (win vs } \\
\text { defeat) }\end{array}$ & $\begin{array}{l}\text { ES (draw vs } \\
\text { defeat) }\end{array}$ \\
\hline DOMS (au) & $\begin{array}{l}3.7 \pm 0.2 \mathrm{a}, \mathrm{b}, \mathrm{c}, \\
\mathrm{d}, \mathrm{e}\end{array}$ & $4.7 \pm 0.2 \mathrm{a}, \mathrm{b}, \mathrm{c}, \mathrm{d}$ & $3.3 \pm 0.3^{\mathrm{e}}$ & $\begin{array}{l}-4.08 \\
(-5.07 \\
-2.92) *\end{array}$ & $\begin{array}{l}1.75(0.99 \\
2.44)\end{array}$ & $\begin{array}{l}5.42(3.99 \\
6.63) *\end{array}$ \\
\hline Sleep (au) & $2.9 \pm 0.2^{\mathrm{d}, \mathrm{e}}$ & $3.3 \pm 0.2 \mathrm{~d}, \mathrm{e}$ & $3.0 \pm 0.2^{\mathrm{e}}$ & $\begin{array}{l}-2.08 \\
(-2.80 \\
-1.28)\end{array}$ & $\begin{array}{l}-0.40 \\
(-1.02,0.23)\end{array}$ & $\begin{array}{l}1.42(0.70, \\
2.08)\end{array}$ \\
\hline $\begin{array}{l}\text { Fatigue } \\
\text { (au) }\end{array}$ & $\begin{array}{l}3.5 \pm 0.3 \text { a, c, d } \\
\text { e }\end{array}$ & $4.6 \pm 0.1 \mathrm{a}, \mathrm{b}, \mathrm{c}, \mathrm{d}$ & $3.5 \pm 0.2 \mathrm{~b}, \mathrm{~d}, \mathrm{e}$ & $\begin{array}{l}-5.17 \\
(-6.33 \\
-3.79) *\end{array}$ & $\begin{array}{l}0.16(-0.46 \\
0.78)\end{array}$ & $\begin{array}{l}5.86(4.34 \\
7.15) *\end{array}$ \\
\hline Stress (au) & $2.5 \pm 0.1$ & $3.0 \pm 0.2$ & $2.6 \pm 0.2$ & $\begin{array}{l}-2.88 \\
(-3.69 \\
-1.94)\end{array}$ & $\begin{array}{l}-1.08 \\
(-1.72 \\
-0.40)\end{array}$ & $\begin{array}{l}1.36(0.65, \\
2.02)\end{array}$ \\
\hline HI (au) & $\begin{array}{l}12.6 \pm 0.7^{\mathrm{c}, \mathrm{d}}, \\
\mathrm{e}\end{array}$ & $15.5 \pm 0.6^{\mathrm{a}, \mathrm{b}, \mathrm{c}, \mathrm{d}}$ & $12.4 \pm 0.7^{\mathrm{e}}$ & $\begin{array}{l}-4.54 \\
(-5.61 \\
-3.30) *\end{array}$ & $\begin{array}{l}0.32(-0.31 \\
0.94)\end{array}$ & $\begin{array}{l}4.59(3.34 \\
5.67) *\end{array}$ \\
\hline $\begin{array}{l}\text { Duration } \\
\text { (min) }\end{array}$ & $\begin{array}{l}56.3 \pm 1.3 \text { a, b, } \\
c, d, e\end{array}$ & $30.3 \pm 1.2^{a, b}, \mathrm{c}, \mathrm{d}, \mathrm{e}$ & $\begin{array}{l}64.9 \pm 2.4^{\mathrm{a}, \mathrm{b}} \\
\mathrm{c}, \mathrm{d}, \mathrm{e}\end{array}$ & $\begin{array}{l}20.98(16.02, \\
25.11) *\end{array}$ & $\begin{array}{l}-4.47 \\
(-5.53 \\
-3.24) *\end{array}$ & $\begin{array}{l}-18.12 \\
(-21.71 \\
-13.82) *\end{array}$ \\
\hline s-RPE (au) & $\begin{array}{l}171.2 \pm 9.2^{\mathrm{a}} \\
\mathrm{b}, \mathrm{c}, \mathrm{d}, \mathrm{e}\end{array}$ & $69.7 \pm 4.6 \mathrm{a}, \mathrm{b}, \mathrm{c}, \mathrm{d}, \mathrm{e}$ & $\begin{array}{l}242.7 \pm 25.4 \mathrm{~b}, \\
\mathrm{e}\end{array}$ & $\begin{array}{l}13.96(10.62, \\
16.75) *\end{array}$ & $\begin{array}{l}-3.74 \\
(-4.68 \\
-2.65) *\end{array}$ & $\begin{array}{l}-9.47(-11.41 \\
-7.16) *\end{array}$ \\
\hline $\begin{array}{l}\text { Total } \\
\text { Distance } \\
\text { (m) }\end{array}$ & $\begin{array}{l}7005.6 \pm 149.3 \\
c, d, e\end{array}$ & $\begin{array}{l}7112.4 \pm 98.5^{\mathrm{a}, \mathrm{c}, \mathrm{d}}, \\
\mathrm{e}\end{array}$ & $\begin{array}{l}7326.1 \pm 83.0 \\
\text { a, c, d, e }\end{array}$ & $\begin{array}{l}-0.84 \\
(-1.47 \\
-0.18)\end{array}$ & $\begin{array}{l}-2.65 \\
(-3.44 \\
-1.76)\end{array}$ & $\begin{array}{l}-2.35(-3.10 \\
-1.50)\end{array}$ \\
\hline $\operatorname{HSRD}(\mathrm{m})$ & $\begin{array}{l}278.9 \pm 21.2^{\mathrm{c}} \\
\mathrm{d}, \mathrm{e}\end{array}$ & $284.1 \pm 18.2 \mathrm{c}, \mathrm{d}, \mathrm{e}$ & $\begin{array}{l}294.5 \pm 24.5^{\mathrm{d}}, \\
\mathrm{e}\end{array}$ & $\begin{array}{l}-0.26 \\
(-0.88,0.36)\end{array}$ & $\begin{array}{l}-0.68 \\
(-1.30 \\
-0.03)\end{array}$ & $\begin{array}{l}-0.48(-1.10 \\
0.16)\end{array}$ \\
\hline $\begin{array}{l}\mathrm{AvS} \\
(\mathrm{m} / \mathrm{min})\end{array}$ & $\begin{array}{l}125.7 \pm 4.2^{\mathrm{a}} \\
\mathrm{b}, \mathrm{c}, \mathrm{d}, \mathrm{e}\end{array}$ & $\begin{array}{l}122.3 \pm 3.5 \mathrm{a}, \mathrm{b}, \mathrm{c}, \mathrm{d} \text {, } \\
\mathrm{e}\end{array}$ & $\begin{array}{l}115.5 \pm 4.9^{\mathrm{a}} \\
\mathrm{b}, \mathrm{c}, \mathrm{d}, \mathrm{e}\end{array}$ & $\begin{array}{l}0.89(0.22, \\
1.52)\end{array}$ & $\begin{array}{l}2.24(1.41 \\
2.99)\end{array}$ & $\begin{array}{l}1.60(0.86 \\
2.28)\end{array}$ \\
\hline
\end{tabular}




\begin{tabular}{|c|c|c|c|c|c|c|}
\hline $\begin{array}{l}\text { MD-4 } \\
(n=20)\end{array}$ & & & & & & \\
\hline DOMS (au) & $2.8 \pm 0.2^{\mathrm{e}}$ & $2.3 \pm 0.2 \mathrm{~b}, \mathrm{e}$ & $2.9 \pm 0.2^{\mathrm{e}}$ & $\begin{array}{l}2.52(1.65 \\
3.29) *\end{array}$ & $\begin{array}{l}-0.61 \\
(-1.24,0.03)\end{array}$ & $\begin{array}{l}-3.08(-3.93, \\
-2.11) *\end{array}$ \\
\hline Sleep (au) & $2.8 \pm 0.1^{\mathrm{e}}$ & $2.7 \pm 0.2^{\mathrm{e}}$ & $3.5 \pm 0.2$ & $\begin{array}{l}0.36(-0.27 \\
0.98)\end{array}$ & $\begin{array}{l}-3.57 \\
(-4.48 \\
-2.51)\end{array}$ & $\begin{array}{l}-3.33(-4.21, \\
-2.32) *\end{array}$ \\
\hline $\begin{array}{l}\text { Fatigue } \\
(\mathrm{au})\end{array}$ & $2.7 \pm 0.2^{\mathrm{e}}$ & $2.3 \pm 0.2 \mathrm{~d}, \mathrm{e}$ & $2.9 \pm 0.2^{\mathrm{e}}$ & $\begin{array}{l}1.87(1.09 \\
2.57) *\end{array}$ & $\begin{array}{l}-0.91 \\
(-1.54 \\
-0.24) *\end{array}$ & $\begin{array}{l}-2.83(-3.64, \\
-1.91) *\end{array}$ \\
\hline Stress (au) & $2.5 \pm 0.2$ & $2.4 \pm 0.2$ & $2.4 \pm 0.2$ & $\begin{array}{l}0.35(-0.28, \\
0.97)\end{array}$ & $\begin{array}{l}0.66(0.01, \\
1.28)\end{array}$ & $\begin{array}{l}0.24(-0.39 \\
0.85)\end{array}$ \\
\hline $\mathrm{HI}(\mathrm{au})$ & $10.8 \pm 0.6^{\mathrm{e}}$ & $9.7 \pm 0.7^{\mathrm{e}}$ & $11.7 \pm 0.6^{\mathrm{e}}$ & $\begin{array}{l}1.69(0.94, \\
2.38)\end{array}$ & $\begin{array}{l}-1.52 \\
(-2.19 \\
-0.79)\end{array}$ & $\begin{array}{l}-3.10(-3.94 \\
-2.13) *\end{array}$ \\
\hline $\begin{array}{l}\text { Duration } \\
(\min )\end{array}$ & $80.8 \pm 0.8 \mathrm{~d}, \mathrm{e}$ & $81.5 \pm 0.5^{c, d, e}$ & $\begin{array}{l}78.8 \pm 1.2 \mathrm{c}, \mathrm{d}, \\
\mathrm{e}\end{array}$ & $\begin{array}{l}-0.89 \\
(-1.52 \\
-0.23)\end{array}$ & $\begin{array}{l}1.89(1.12, \\
2.60)\end{array}$ & $\begin{array}{l}2.75(1.84, \\
3.55)\end{array}$ \\
\hline s-RPE (au) & $\begin{array}{l}361.0 \pm 27.3^{c} \\
d, e\end{array}$ & $404.2 \pm 31.3^{c, d, e}$ & $\begin{array}{l}360.7 \pm 26.9 \mathrm{~d} \\
\mathrm{e}\end{array}$ & $\begin{array}{l}-1.47 \\
(-2.14 \\
-0.74)\end{array}$ & $\begin{array}{l}0.01(-0.61, \\
0.63)\end{array}$ & $\begin{array}{l}1.49(0.76, \\
2.16)\end{array}$ \\
\hline $\begin{array}{l}\text { Total } \\
\text { Distance } \\
\text { (m) }\end{array}$ & $\begin{array}{l}6467.9 \pm 90.2 \\
c, d, e\end{array}$ & $6374.9 \pm 83.0 \mathrm{c}, \mathrm{d}, \mathrm{e}$ & $\begin{array}{l}6186.5 \pm 109.6 \\
b, d, e\end{array}$ & $\begin{array}{l}1.08(0.40, \\
1.72)\end{array}$ & $\begin{array}{l}2.80(1.88, \\
3.61)\end{array}$ & $\begin{array}{l}1.93(1.15, \\
2.64)\end{array}$ \\
\hline $\operatorname{HSRD}(\mathrm{m})$ & $\begin{array}{l}290.7 \pm 17.8^{c} \\
d, e\end{array}$ & $273.8 \pm 15.4 \mathrm{c}, \mathrm{d}, \mathrm{e}$ & $\begin{array}{l}240.0 \pm 13.5^{b} \\
d, e\end{array}$ & $\begin{array}{l}1.01(0.34, \\
1.65) *\end{array}$ & $\begin{array}{l}3.21(2.22 \\
4.07) *\end{array}$ & $\begin{array}{l}2.33(1.49 \\
3.08) *\end{array}$ \\
\hline $\begin{array}{l}\mathrm{AvS} \\
(\mathrm{m} / \mathrm{min})\end{array}$ & $\begin{array}{l}80.1 \pm 1.2 \mathrm{c}, \mathrm{d}, \\
\mathrm{e}\end{array}$ & $79.6 \pm 1.1 \mathrm{~d}, \mathrm{e}$ & $\begin{array}{l}78.7 \pm 1.5 \mathrm{~b}, \mathrm{c}, \\
\mathrm{d}, \mathrm{e}\end{array}$ & $\begin{array}{l}0.42(-0.21, \\
1.04)\end{array}$ & $\begin{array}{l}1.07(0.39, \\
1.71)\end{array}$ & $\begin{array}{l}0.73(0.07 \\
1.35)\end{array}$ \\
\hline \multicolumn{7}{|l|}{$\begin{array}{l}\text { MD-3 } \\
(n=24)\end{array}$} \\
\hline DOMS (au) & $2.9 \pm 0.2^{\mathrm{e}}$ & $3.0 \pm 0.2^{\mathrm{e}}$ & $3.0 \pm 0.2^{\mathrm{e}}$ & $\begin{array}{l}-0.66 \\
(-1.28 \\
-0.01)\end{array}$ & $\begin{array}{l}-0.94 \\
(-1.57 \\
-0.27)\end{array}$ & $\begin{array}{l}-0.31(-0.93, \\
0.32)\end{array}$ \\
\hline Sleep (au) & $2.912 \pm 0.2^{\mathrm{e}}$ & $3.1 \pm 0.1 \mathrm{~d}, \mathrm{e}$ & $3.3 \pm 0.2^{\mathrm{e}}$ & $\begin{array}{l}-1.56 \\
(-2.23 \\
-0.82)\end{array}$ & $\begin{array}{l}-2.20 \\
(-2.94 \\
-1.38)\end{array}$ & $\begin{array}{l}-0.78(-1.41, \\
-0.13)\end{array}$ \\
\hline $\begin{array}{l}\text { Fatigue } \\
(\mathrm{au})\end{array}$ & $2.9 \pm 0.2^{\mathrm{e}}$ & $2.9 \pm 0.2^{\mathrm{e}}$ & $3.0 \pm 0.2^{\mathrm{e}}$ & $\begin{array}{l}-0.16 \\
(-0.78,0.46)\end{array}$ & $\begin{array}{l}-0.22 \\
(-0.84,0.40)\end{array}$ & $\begin{array}{l}-0.07(-0.69, \\
0.55)\end{array}$ \\
\hline Stress (au) & $2.5 \pm 0.2$ & $2.4 \pm 0.2$ & $2.4 \pm 0.2$ & $\begin{array}{l}0.44(-0.20, \\
1.06)\end{array}$ & $\begin{array}{l}0.63(-0.02, \\
1.25)\end{array}$ & $\begin{array}{l}0.21(-0.42, \\
0.82)\end{array}$ \\
\hline $\mathrm{HI}(\mathrm{au})$ & $11.1 \pm 0.6^{\mathrm{e}}$ & $11.4 \pm 0.5^{\mathrm{e}}$ & $11.6 \pm 0.6^{\mathrm{e}}$ & $\begin{array}{l}-0.58 \\
(-1.20,0.06)\end{array}$ & $\begin{array}{l}-0.83 \\
(-1.45 \\
-0.17)\end{array}$ & $\begin{array}{l}-0.28(-0.90, \\
0.35)\end{array}$ \\
\hline
\end{tabular}




\begin{tabular}{|c|c|c|c|c|c|c|}
\hline $\begin{array}{l}\text { Duration } \\
\text { (min) }\end{array}$ & $79.9 \pm 0.9 \mathrm{~d}, \mathrm{e}$ & $77.5 \pm 1.4 \mathrm{c}, \mathrm{d}, \mathrm{e}$ & $\begin{array}{l}76.3 \pm 1.8^{c, d} \\
e\end{array}$ & $\begin{array}{l}2.04(1.24, \\
2.76)\end{array}$ & $\begin{array}{l}2.47(1.61 \\
3.24)\end{array}$ & $\begin{array}{l}0.73(0.07 \\
1.35)\end{array}$ \\
\hline s-RPE (au) & $\begin{array}{l}375.8 \pm 26.2^{c} \\
d, e\end{array}$ & $365.7 \pm 24.0^{c, d, e}$ & $\begin{array}{l}360.7 \pm 26.1 \mathrm{~d} \\
\mathrm{e}\end{array}$ & $\begin{array}{l}0.40(-0.23, \\
1.02)\end{array}$ & $\begin{array}{l}0.58(-0.07 \\
1.20)\end{array}$ & $\begin{array}{l}0.20(-0.42 \\
0.82)\end{array}$ \\
\hline $\begin{array}{l}\text { Total } \\
\text { Distance } \\
\text { (m) }\end{array}$ & $\begin{array}{l}6723.6 \pm 102.4 \\
c, d, e\end{array}$ & $6699.6 \pm 12.6^{\mathrm{c}, \mathrm{d}, \mathrm{e}}$ & $\begin{array}{l}7108.5 \pm 141.7 \\
c, d, e\end{array}$ & $\begin{array}{l}0.21(-0.42 \\
0.82)\end{array}$ & $\begin{array}{l}-3.11 \\
(-3.96 \\
-2.14) *\end{array}$ & $\begin{array}{l}-3.01(-3.85 \\
-2.06) *\end{array}$ \\
\hline $\operatorname{HSRD}(\mathrm{m})$ & $\begin{array}{l}240.7 \pm 15.7 \mathrm{~d} \\
\mathrm{e}\end{array}$ & $233.0 \pm 22.3^{\mathrm{c}, \mathrm{d}, \mathrm{e}}$ & $\begin{array}{l}307.3 \pm 15.0^{\mathrm{c}} \\
\mathrm{d}, \mathrm{e}\end{array}$ & $\begin{array}{l}0.40(-0.23 \\
1.02)\end{array}$ & $\begin{array}{l}-4.34 \\
(-5.37 \\
-3.13) *\end{array}$ & $\begin{array}{l}-3.91(-4.88 \\
-2.79) *\end{array}$ \\
\hline $\begin{array}{l}\mathrm{AvS} \\
(\mathrm{m} / \mathrm{min})\end{array}$ & $\begin{array}{l}84.2 \pm 1.0^{\mathrm{c}, \mathrm{d}} \\
\mathrm{e}\end{array}$ & $87.0 \pm 2.6^{\mathrm{d}, \mathrm{e}}$ & $\begin{array}{l}94.1 \pm 3.2 \mathrm{c}, \mathrm{d}, \\
\mathrm{e}\end{array}$ & $\begin{array}{l}-1.40 \\
(-2.06 \\
-0.68)\end{array}$ & $\begin{array}{l}-4.13 \\
(-5.13 \\
-2.96) *\end{array}$ & $\begin{array}{l}-2.44(-3.20, \\
-1.58) *\end{array}$ \\
\hline \multicolumn{7}{|l|}{$\begin{array}{l}\text { MD-2 } \\
(n=24)\end{array}$} \\
\hline DOMS (au) & $3.0 \pm 0.2^{\mathrm{e}}$ & $3.100 \pm 0.205^{\mathrm{e}}$ & $3.1 \pm 0.2^{\mathrm{e}}$ & $\begin{array}{l}-0.49 \\
(-1.11,0.14)\end{array}$ & $\begin{array}{l}-0.43 \\
(-1.05,0.21)\end{array}$ & $\begin{array}{l}0.08(-0.54 \\
0.70)\end{array}$ \\
\hline Sleep (au) & $2.7 \pm 0.2^{\mathrm{e}}$ & $2.633 \pm 0.154^{\mathrm{e}}$ & $2.9 \pm 0.2^{\mathrm{e}}$ & $\begin{array}{l}0.20(-0.42, \\
0.82)\end{array}$ & $\begin{array}{l}-1.71 \\
(-2.40 \\
-0.96)\end{array}$ & $\begin{array}{l}-1.98(-2.69, \\
-1.19)\end{array}$ \\
\hline $\begin{array}{l}\text { Fatigue } \\
\text { (au) }\end{array}$ & $2.9 \pm 0.2^{\mathrm{e}}$ & $2.950 \pm 0.257^{\mathrm{e}}$ & $2.9 \pm 0.2^{\mathrm{e}}$ & $\begin{array}{l}-0.03 \\
(-0.65,0.59)\end{array}$ & $\begin{array}{l}0.06(-0.56 \\
0.68)\end{array}$ & $\begin{array}{l}0.09(-0.53 \\
0.71)\end{array}$ \\
\hline Stress (au) & $2.5 \pm 0.2$ & $2.917 \pm 0.219$ & $2.5 \pm 0.2$ & $\begin{array}{l}-1.91 \\
(-2.61 \\
-1.13)\end{array}$ & $\begin{array}{l}0.08(-0.54 \\
0.70)\end{array}$ & $\begin{array}{l}1.97(1.18, \\
2.68)\end{array}$ \\
\hline $\mathrm{HI}(\mathrm{au})$ & $11.1 \pm 0.6^{\mathrm{e}}$ & $11.600 \pm 0.725^{\mathrm{e}}$ & $11.6 \pm 0.6^{\mathrm{e}}$ & $\begin{array}{l}-0.68 \\
(-1.30 \\
-0.03)\end{array}$ & $\begin{array}{l}-0.85 \\
(-1.48 \\
-0.19)\end{array}$ & $\begin{array}{l}-0.07(-0.69, \\
0.55)\end{array}$ \\
\hline $\begin{array}{l}\text { Duration } \\
\text { (min) }\end{array}$ & $77.9 \pm 0.5^{\mathrm{d}, \mathrm{e}}$ & $\begin{array}{l}69.017 \pm 00.728 \mathrm{~d} \\
\mathrm{e}\end{array}$ & $88.1 \pm 1.5^{\mathrm{e}}$ & $\begin{array}{l}14.46(11.01 \\
17.34) *\end{array}$ & $\begin{array}{l}-8.93 \\
(-10.77 \\
-6.74) *\end{array}$ & $\begin{array}{l}-15.79 \\
(-18.93 \\
-12.03) *\end{array}$ \\
\hline s-RPE (au) & $\begin{array}{l}310.8 \pm 23.0^{\mathrm{d}} \\
\mathrm{e}\end{array}$ & $223.217 \pm 14.966^{\mathrm{e}}$ & $\begin{array}{l}340.1 \pm 20.4^{d}, \\
\text { e }\end{array}$ & $\begin{array}{l}4.52(3.27 \\
5.58) *\end{array}$ & $\begin{array}{l}-1.35 \\
(-2.01 \\
-0.64)\end{array}$ & $\begin{array}{l}-6.54(-7.94 \\
-4.87) *\end{array}$ \\
\hline $\begin{array}{l}\text { Total } \\
\text { Distance } \\
\text { (m) }\end{array}$ & $\begin{array}{l}5649.4 \pm 58.3 \\
d, e\end{array}$ & $\begin{array}{l}5577.370 \pm 137.593 \\
d, e\end{array}$ & $\begin{array}{l}6109.3 \pm 88.5 \\
d, e\end{array}$ & $\begin{array}{l}0.68(0.03, \\
1.30)\end{array}$ & $\begin{array}{l}-6.14 \\
(-7.47 \\
-4.56) *\end{array}$ & $\begin{array}{l}-4.60(-5.67 \\
-3.34) *\end{array}$ \\
\hline HSRD (m) & $\begin{array}{l}216.2 \pm 10.8^{d} \\
\text { e }\end{array}$ & $160.054 \pm 18.668^{e}$ & $\begin{array}{l}225.7 \pm 16.0 \mathrm{~d} \\
\mathrm{e}\end{array}$ & $\begin{array}{l}3.68(2.60 \\
4.61) *\end{array}$ & $\begin{array}{l}-0.69 \\
(-1.32 \\
-0.04)\end{array}$ & $\begin{array}{l}-3.77(-4.72, \\
-2.68) *\end{array}$ \\
\hline $\begin{array}{l}\mathrm{AvS} \\
(\mathrm{m} / \mathrm{min})\end{array}$ & $72.5 \pm 0.8^{\mathrm{d}, \mathrm{e}}$ & $81.227 \pm 1.988^{\mathrm{d}, \mathrm{e}}$ & $69.6 \pm 1.3^{\mathrm{d}, \mathrm{e}}$ & $\begin{array}{l}-5.76 \\
(-7.02 \\
-4.26) *\end{array}$ & $\begin{array}{l}2.65(1.76 \\
3.44)\end{array}$ & $\begin{array}{l}6.87(5.13 \\
8.33) *\end{array}$ \\
\hline
\end{tabular}




\begin{tabular}{|c|c|c|c|c|c|c|}
\hline $\begin{array}{l}\text { MD-1 } \\
(n=24)\end{array}$ & & & & & & \\
\hline DOMS (au) & $3.0 \pm 0.2^{\mathrm{e}}$ & $3.0 \pm 0.281^{\mathrm{e}}$ & $2.9 \pm 0.2^{\mathrm{e}}$ & $\begin{array}{l}-0.06 \\
(-0.68,0.56)\end{array}$ & $\begin{array}{l}0.33(-0.30 \\
0.95)\end{array}$ & $\begin{array}{l}0.32(-0.31 \\
0.93)\end{array}$ \\
\hline Sleep (au) & $2.6 \pm 0.2^{\mathrm{e}}$ & $2.3 \pm 0.149^{\mathrm{e}}$ & $2.9 \pm 0.1 \mathrm{e}$ & $\begin{array}{l}1.93(1.14, \\
2.63)\end{array}$ & $\begin{array}{l}-2.03 \\
(-2.75 \\
-1.23) *\end{array}$ & $\begin{array}{l}-4.27(-5.30, \\
-3.08) *\end{array}$ \\
\hline $\begin{array}{l}\text { Fatigue } \\
\text { (au) }\end{array}$ & $2.8 \pm 0.2^{\mathrm{e}}$ & $2.8 \pm 0.259^{\mathrm{e}}$ & $2.9 \pm 0.2^{\mathrm{e}}$ & $\begin{array}{l}0.18(-0.45, \\
0.80)\end{array}$ & $\begin{array}{l}0.06(-0.56, \\
0.68)\end{array}$ & $\begin{array}{l}-0.12(-0.74, \\
0.50)\end{array}$ \\
\hline Stress (au) & $2.5 \pm 0.2$ & $2.5 \pm 0.297$ & $2.5 \pm 0.2$ & $\begin{array}{l}0.08(-0.54, \\
0.70)\end{array}$ & $\begin{array}{l}-0.04 \\
(-0.66,0.58)\end{array}$ & $\begin{array}{l}-0.11(-0.73, \\
0.51)\end{array}$ \\
\hline HI (au) & $10.9 \pm 0.6^{\mathrm{e}}$ & $10.6 \pm 0.606^{\mathrm{e}}$ & $11.2 \pm 0.6^{\mathrm{e}}$ & $\begin{array}{l}0.60(-0.04, \\
1.22)\end{array}$ & $\begin{array}{l}-0.43 \\
(-1.05,0.21)\end{array}$ & $\begin{array}{l}-1.00(-1.64, \\
-0.32)\end{array}$ \\
\hline $\begin{array}{l}\text { Duration } \\
\text { (min) }\end{array}$ & $84.647 \pm 0.5^{\mathrm{e}}$ & $95.1 \pm 1.500^{\mathrm{e}}$ & $91.5 \pm 1.6^{\mathrm{e}}$ & $\begin{array}{l}-9.37 \\
(-11.29 \\
-7.08) *\end{array}$ & $\begin{array}{l}-5.80 \\
(-7.08 \\
-4.29) *\end{array}$ & $\begin{array}{l}2.31(1.47 \\
3.05)\end{array}$ \\
\hline s-RPE (au) & $214.4 \pm 13.5^{\mathrm{e}}$ & $211.1 \pm 15.986^{\mathrm{e}}$ & $206.6 \pm 25.6^{\mathrm{e}}$ & $\begin{array}{l}0.22(-0.40, \\
0.84)\end{array}$ & $\begin{array}{l}0.38(-0.25, \\
1.00)\end{array}$ & $\begin{array}{l}0.21(-0.42 \\
0.83)\end{array}$ \\
\hline $\begin{array}{l}\text { Total } \\
\text { Distance } \\
\text { (m) }\end{array}$ & $\begin{array}{l}3628.6 \pm 57.2 \\
\mathrm{e}\end{array}$ & $3391.3 \pm 153.278^{\mathrm{e}}$ & $\begin{array}{l}3236.1 \pm 113.7 \\
\mathrm{e}\end{array}$ & $\begin{array}{l}2.05(1.25 \\
2.77)\end{array}$ & $\begin{array}{l}4.36(3.15 \\
5.40) *\end{array}$ & $\begin{array}{l}1.15(0.46, \\
1.79)\end{array}$ \\
\hline HSRD (m) & $86.0 \pm 6.9^{\mathrm{e}}$ & $130.7 \pm 17.567$ & $54.8 \pm 7.1$ & $\begin{array}{l}-3.35 \\
(-4.23 \\
-2.34)\end{array}$ & $\begin{array}{l}4.47(3.24 \\
5.53) *\end{array}$ & $\begin{array}{l}5.67(4.19 \\
6.92) *\end{array}$ \\
\hline $\begin{array}{l}\mathrm{AvS} \\
(\mathrm{m} / \mathrm{min})\end{array}$ & $42.8 \pm 0.6^{\mathrm{e}}$ & $36.1 \pm 1.700^{\mathrm{e}}$ & $35.8 \pm 1.6^{\mathrm{e}}$ & $\begin{array}{l}5.34(3.93 \\
6.53) *\end{array}$ & $\begin{array}{l}5.75(4.25 \\
7.01) *\end{array}$ & $\begin{array}{l}0.17(-0.46 \\
0.79) *\end{array}$ \\
\hline $\begin{array}{l}\text { MD+1 } \\
(n=20)\end{array}$ & & & & & & \\
\hline DOMS (au) & $4.3 \pm 0.3$ & $4.8 \pm 0.1$ & $4.4 \pm 0.3$ & $\begin{array}{l}-2.63 \\
(-3.42 \\
-1.74)\end{array}$ & $\begin{array}{l}-0.32 \\
(-0.94,0.31)\end{array}$ & $\begin{array}{l}2.09(1.28 \\
2.81)\end{array}$ \\
\hline Sleep (au) & $3.8 \pm 0.2$ & $4.0 \pm 0.1$ & $4.4 \pm 0.1$ & $\begin{array}{l}-1.29 \\
(-1.94 \\
-0.58)\end{array}$ & $\begin{array}{l}-3.40 \\
(-4.29 \\
-2.37)\end{array}$ & $\begin{array}{l}-3.55(-4.46 \\
-2.50)\end{array}$ \\
\hline $\begin{array}{l}\text { Fatigue } \\
\text { (au) }\end{array}$ & $4.3 \pm 0.3$ & $4.9 \pm 0.1$ & $4.5 \pm 0.3$ & $\begin{array}{l}-2.92 \\
(-3.75 \\
-1.98)\end{array}$ & $\begin{array}{l}-0.61 \\
(-1.23,0.03)\end{array}$ & $\begin{array}{l}2.02(1.22 \\
2.73)\end{array}$ \\
\hline Stress (au) & $26 \pm 0.2$ & $3.5 \pm 0.2$ & $2.8 \pm 0.2$ & $\begin{array}{l}-4.54 \\
(-5.61 \\
-3.30)\end{array}$ & $\begin{array}{l}-0.92 \\
(-1.55 \\
-0.25)\end{array}$ & $\begin{array}{l}3.36(2.34 \\
4.25) *\end{array}$ \\
\hline HI (au) & $15.0 \pm 0.7$ & $17.3 \pm 0.4$ & $16.1 \pm 0.7$ & $\begin{array}{l}-3.86 \\
(-4.82 \\
-2.75) *\end{array}$ & $\begin{array}{l}-1.55 \\
(-2.22 \\
-0.81)\end{array}$ & $\begin{array}{l}2.21(1.39 \\
2.95)\end{array}$ \\
\hline
\end{tabular}




\begin{tabular}{|c|c|c|c|c|c|c|}
\hline $\begin{array}{l}\text { Duration } \\
\text { (min) }\end{array}$ & $18.6 \pm 1.1$ & $15.0 \pm 0.0$ & $16.8 \pm 1.4$ & $\begin{array}{l}4.64(3.37 \\
5.72) *\end{array}$ & $\begin{array}{l}1.42(0.70, \\
2.08)\end{array}$ & $\begin{array}{l}-1.76(-2.46, \\
-1.00)\end{array}$ \\
\hline s-RPE (au) & $33.2 \pm 4.5$ & $32.3 \pm 1.9$ & $32.9 \pm 4.1$ & $\begin{array}{l}0.26(-0.37 \\
0.88) *\end{array}$ & $\begin{array}{l}0.06(-0.56, \\
0.68)\end{array}$ & $\begin{array}{l}-0.21(-0.82 \\
0.42)\end{array}$ \\
\hline $\begin{array}{l}\text { Total } \\
\text { Distance } \\
\text { (m) }\end{array}$ & $4418.7 \pm 68.3$ & $4394.2 \pm 61.5$ & $4345.2 \pm 108.3$ & $\begin{array}{l}0.38(-0.26 \\
0.99)\end{array}$ & $\begin{array}{l}0.81(0.15, \\
1.44)\end{array}$ & $\begin{array}{l}0.56(-0.09 \\
1.18)\end{array}$ \\
\hline $\operatorname{HSRD}(\mathrm{m})$ & $109.4 \pm 10.6$ & $104.8 \pm 10.1$ & $95.5 \pm 11.1$ & $\begin{array}{l}0.45(-0.19, \\
1.07)\end{array}$ & $\begin{array}{l}1.29(0.58, \\
1.94)\end{array}$ & $\begin{array}{l}0.88(0.21, \\
1.51)\end{array}$ \\
\hline $\begin{array}{l}\mathrm{AvS} \\
(\mathrm{m} / \mathrm{min})\end{array}$ & $249.9 \pm 12.7$ & $258.9 \pm 9.9$ & $277.0 \pm 13.4$ & $\begin{array}{l}-0.79 \\
(-1.42 \\
-0.13)\end{array}$ & $\begin{array}{l}-2.07 \\
(-2.80 \\
-1.27)\end{array}$ & $\begin{array}{l}-1.53(-2.20, \\
-0.80)\end{array}$ \\
\hline
\end{tabular}

MD-=matchday minus $(5,4,3,2,1)$; MD+1= matchday plus 1 ; DOMS = delayed onset muscle soreness; au=arbitrary units; $\mathrm{HI}=$ Hooper index; $\min =$ minutes; $m=$ meters; s-RPE= session rating of perceived exertion; HSRD = high-speed running distance; $\mathrm{AvS}=$ average speed.

a denotes difference from MD-4, $b$ denotes difference from MD-3, c denotes difference from MD-2, d denotes difference from MD-1, e denotes difference from MD+1, all $p<0.05$.

* significant differences between win vs draw; win vs defeat; draw vs defeat, respectively by column $(p<0.01)$.

We analysed physical performance for the weeks that preceded $78.43 \%$ wins, $5.88 \%$ draws and $15.69 \%$ defeats over the entire season.

\subsection{Comparison of TL from training sessions preceding wins, draws or defeats results}

The main results demonstrated that MD-1 displayed significantly lower values than any other day for total distance, average speed and high-speed running distance, all external TL variables. In opposition, internal TL represented by s-RPE showed lower values in MD+1 and Hooper Index showed higher values in MD +1 . These results were similar in any kind of result situation (win, draw and defeat). In addition, Table 2 presents differences between starters vs non-starters regarding $\mathrm{MD}+1$ and only stress showed significant difference between them $(p<0.05)$ starters and non-starters.

alt-text: Table 2

Table 2

(i) The table layout displayed in this section is not how it will appear in the final version. The representation below is solely purposed for providing corrections to the table. To preview the actual presentation of the table, please view the Proof.

Comparison of match day plus 1 for Training Load Data between wins, draws and defeat for starters and non-starters squad average, Mean \pm SD.

\begin{tabular}{|c|c|c|c|}
\hline MD+1 (starter), $n=10$ & Win & Draw & Defeat \\
\hline DOMS (au) & $4.6 \pm 0.3$ & $4.8 \pm 0.2$ & $4.8 \pm 0.3$ \\
\hline Sleep (au) & $3.7 \pm 0.7$ & $4.1 \pm 1.1$ & $4.4 \pm 0.6$ \\
\hline Fatigue (au) & $4.6 \pm 0.3$ & $4.9 \pm 0.8$ & $4.8 \pm 1.0$ \\
\hline
\end{tabular}




\begin{tabular}{|c|c|c|c|}
\hline Stress (au) & $2.6 \pm 1.0^{\mathrm{a}}$ & $3.8 \pm 1.4$ & $2.9 \pm 1.1$ \\
\hline $\mathrm{HI}(\mathrm{au})$ & $15.6 \pm 2.5$ & $17.5 \pm 1.0$ & $16.8 \pm 0.9$ \\
\hline Duration (min) & $20.6 \pm 1.4$ & $15.0 \pm 0.0$ & $17.3 \pm 2.2$ \\
\hline s-RPE (au) & $40.3 \pm 25.1$ & $28.5 \pm 11.6$ & $32.3 \pm 23.1$ \\
\hline Total Distance (m) & $4407.0 \pm 295.0$ & $4397.3 \pm 213.3$ & $4377.8 \pm 328.6$ \\
\hline $\operatorname{HSRD}(\mathrm{m})$ & $111.0 \pm 34.2$ & $97.1 \pm 26.7$ & $69.4 \pm 23.7$ \\
\hline $\operatorname{AvS}(\mathrm{m} / \mathrm{min})$ & $227.6 \pm 64.0$ & $243.0 \pm 63.9$ & $273.8 \pm 21.2$ \\
\hline \multicolumn{4}{|c|}{ MD+1 (non-starter), $n=10$} \\
\hline DOMS (au) & $4.0 \pm 0.3$ & $4.8 \pm 0.2$ & $4.1 \pm 0.3$ \\
\hline Sleep $(\mathrm{au})$ & $3.8 \pm 1.4$ & $4.0 \pm 0.9$ & $4.5 \pm 0.5$ \\
\hline Fatigue (au) & $3.9 \pm 1.1$ & $4.9 \pm 0.3$ & $4.2 \pm 1.2$ \\
\hline Stress (au) & $2.6 \pm 1.1$ & $3.5 \pm 0.8$ & $2.8 \pm 1.0$ \\
\hline $\mathrm{HI}(\mathrm{au})$ & $14.4 \pm 4.3$ & $17.1 \pm 1.0$ & $15.6 \pm 3.4$ \\
\hline Duration (min) & $16.6 \pm 1.4$ & $15.0 \pm 0.0$ & $17.2 \pm 2.2$ \\
\hline s-RPE (au) & $26.3 \pm 11.8$ & $34.5 \pm 7.9$ & $32.9 \pm 11.3$ \\
\hline Total Distance (m) & $4491.4 \pm 490.9$ & $4432.1 \pm 483.1$ & $4313.7 \pm 676.9$ \\
\hline $\operatorname{HSRD}(\mathrm{m})$ & $112.5 \pm 70.5$ & $114.1 \pm 70.4$ & $117.5 \pm 77.3$ \\
\hline $\operatorname{AvS}(\mathrm{m} / \mathrm{min})$ & $276.4 \pm 52.9$ & $275.6 \pm 48.5$ & $273.8 \pm 21.1$ \\
\hline
\end{tabular}

$\mathrm{MD}+1=$ matchday plus 1; DOMS = delayed onset muscle soreness; au=arbitrary units; $\mathrm{HI}=$ Hooper index; $\min =$ minutes; $m=$ =meters; s-RPE = session rating of perceived exertion; HSRD = high-speed running distance; AvS = average speed.

\section{Table Footnotes}

a denotes difference from MD+1 non-starter, all $P<0.05 .{ }^{*}$ significant differences between win vs draw; win vs defeat; draw vs defeat, respectively by column $(p<0.01)$.

\subsection{Comparison of match day minus for TL data between wins vs draws; wins vs defeats; and draws vs defeats for squad average}

For MD-5, scores from fatigue and DOMS were higher in draws than wins or defeats. Duration of training sessions and s-RPE revealed differences for all match scores, but lower for draws. External TL variables did not present any differences (see Fig. 2).

For MD-4, scores from HI were lower in draws than wins or defeats because in MD-5, general TL were reduced. There were no significant differences for duration of training sessions or s-RPE (see Fig. 1). External TL, especially HSRD, showed a higher value in wins than draws or defeats. In addition, total distance and AVS did not show any differences (see Fig. 2). 


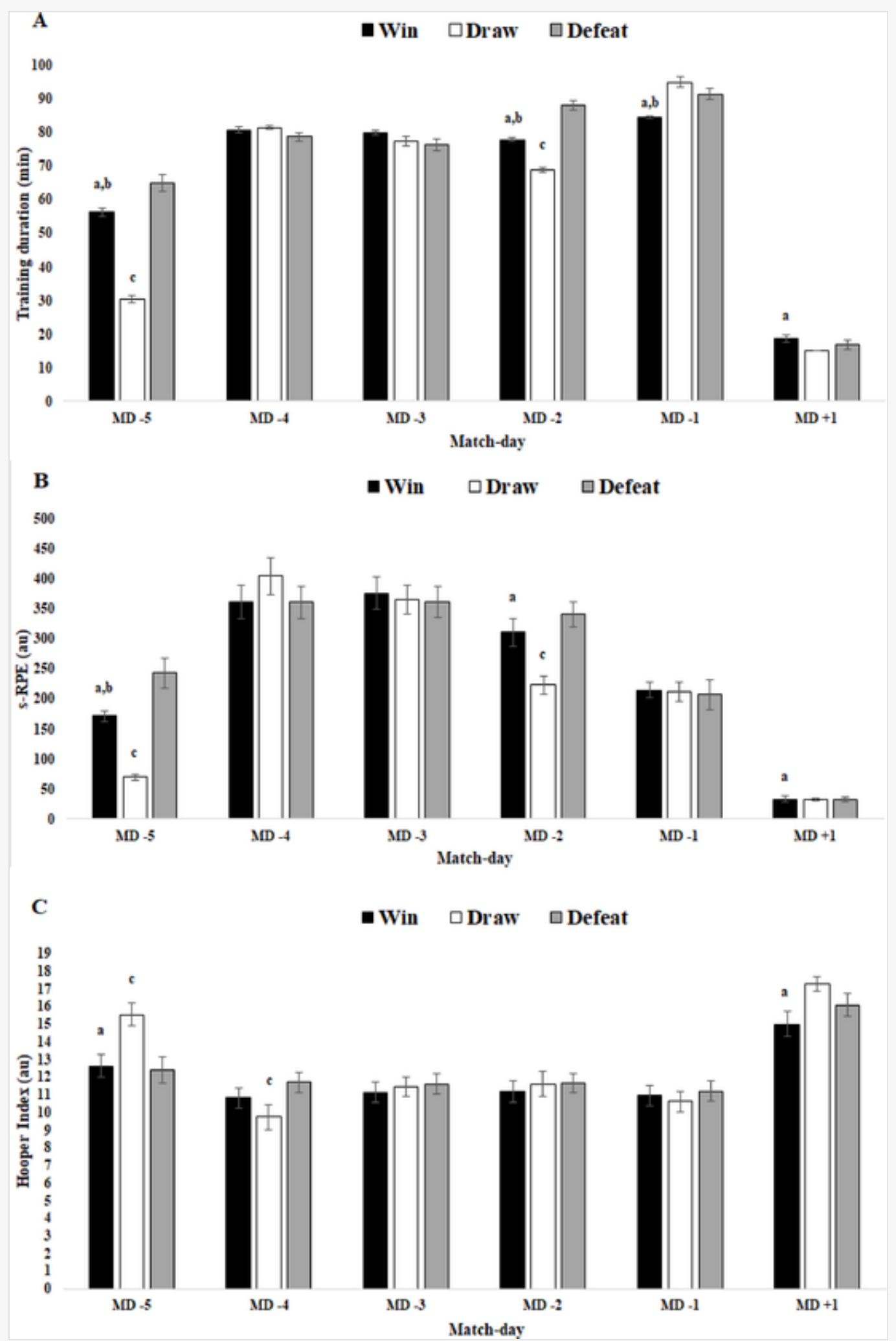

Comparisons between 3 match results for (A) training duration, (B) s-RPE, C) HI. a denotes significant differences between win vs defeat; $\mathrm{b}$ denotes difference between win vs draw; $\mathrm{c}$ denotes significant difference between draw vs defeat, all $(p<0.01)$.

alt-text: Fig 2

Fig. 2 


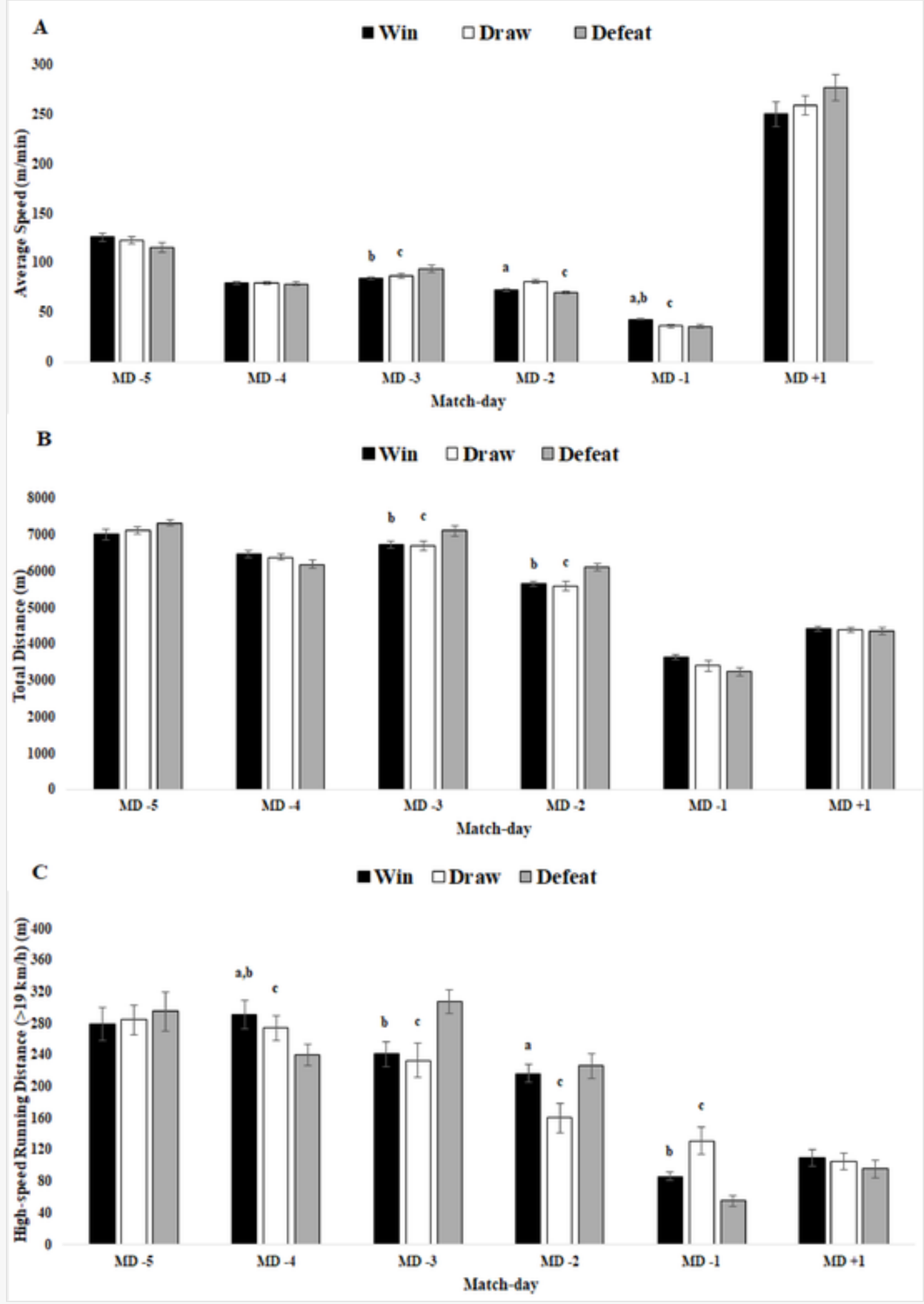

Comparisons between 3 match results for (A) Average Speed, (B) Total Distance, (C) High-speed Running Distance. a denotes significant differences between win vs defeat; b denotes difference between win vs draw; c denotes significant difference between draw vs defeat, all $(p<0.01)$.

For MD-3, scores from HI showed a small increase because TL also increased in MD-4. There were no significant differences for duration of training sessions or s-RPE. HSRD and total distance showed higher distance covered in defeat $>$ win $>$ draw; however, AvS showed higher values in defeat $>$ draw $>$ win (see Fig. 2 ) .

For MD-2, scores from HI remained constant, with no difference between match score. There were more minutes during training sessions, s-RPE and total distance for defeat $>$ win $>$ draw, but AVS showed an inverse 
pattern of significant difference (draw $>$ win $>$ defeat) (see Fig. 2).

For MD-1, scores from HI remained constant with little decrease with no difference between match score. There were more minutes during training sessions in draw $>$ defeat $>$ win, but s-RPE did not show significant differences. External TL total distance did show significant differences, but HSRD were higher in draw $>$ win $>$ defeat and AVS in win $>$ draw $>$ defeat (see Fig. 2).

For $\mathrm{MD}+1$, scores from $\mathrm{HI}$ were higher than all MD- $(5,4,3,2,1)$ and there were significant differences between win vs draw but none vs defeat (see Fig. 1). There were more minutes during training sessions in win $>$ draw $>$ defeat. There were no significant differences for s-RPE or for external TL variables.

\section{Discussion}

The purpose of the present study was to quantify the internal and external TL in a sample of elite European soccer players during the in-season comparing match day minus for TL preceding a win, draw or defeats and comparing TL preceding win versus TL preceding draw or TL preceding defeats.

\subsection{Comparison of TL from training sessions preceding wins, draws or defeats results}

The present study showed higher values of total distance, HSRD and AvS in MD-5 and s-RPE demonstrated a completely different pattern, being higher on MD-4 or MD-3 depending on the result situation (see Table 1). However, when analysing TL in respect to the number of days prior to a match in elite English Premier League players throughout the in-season, Malone et al. [16] found that TL remained similar across all days except for MD-1 in which the load was significantly reduced. In addition, Owen et al. [17] reported that on MD-3, players increased their external TL variables and s-RPE more than other MD- (4, 2 and 1). A plausible justification for our different results patterns in s-RPE could be the decision to reduce, increase or maintain a given power output of the player, which is based on the idea that when a perceived "payoff" is potentially large (know the exercise duration), an athlete would be more likely to incur more homoeostasis disruption [27].

In relation to match-day-minus training comparison, TL increased progressively until MD-3 and later decreased up to MD-1. Similarly, Malone et al. [16] also found a noticeable consistent variation in TL (overall s-RPE and total distance covered) on MD-1 in elite soccer players, when the load was significantly reduced in comparison with the rest of the training days.

In addition, HI scores revealed no variation in days prior to the match day, and so corroborate the results reported by Haddad et al. [11] who suggested that fatigue, stress, DOMS and sleep are not major contributors of perceived exertion during traditional soccer training without excessive TL. In contrast, Clemente et al. [12] found that the relationship between s-RPE and HI revealed significant and negative small-to-moderate correlations during the weeks with two official matches but not during the weeks with one match.

Finally, MD+1 revealed significant results despite the limited training duration $(\sim 20 \mathrm{~min})$. For external TL, average speed has higher values than all other match days' minus. HSRD has higher values than MD-1. However, for internal TL, s-RPE showed a lower value than all other match days (33.6 au). These results may be associated with a high-intensity training session for players who played less minutes or were non-starters. It is also important to note that in-season match-day-minus training comparison was analysed by mean values and microcycles/weeks (7-day period) have different patterns (e.g., some microcycles had training day after the match day and some did not. On the other hand, HI has the higher value than all other match days (15 au, see 
Table 1) and it is relevant to notice that it was recorded before $\mathrm{MD}+1$ begins, which mean that the values are related to the previous match.

As reported above, the s-RPE score resulted either more strongly related to Edward-TL and Banister than training impulse as gold standards when assessing TLs [15]. This occurrence once again supports the findings that s-RPE is a reliable method to characterize training responses $[15,24,25]$. However, our results reveal that sRPE and external TL variables did not follow the same pattern. It seems that RPE may be a physiological and volatile construct that could be different according to the cognitive focus of the player [28]. In addition, Renfree et al. [27] reported that RPE can be an oversimplification of the psychophysiological perceived exertion and a non-conclusive measure for capturing a wide range of sensations experience because RPE can be dissociated from the physiological process through a variety of psychological mechanisms. Another major point and a possible recommendation for future research is to collect RPE during different phases of the training session rather than only 30 min following the end of the training session [29]. These arguments may justify the fact that there were no differences in s-RPE between training days.

\subsection{Comparison of match day minus for TL data between wins vs draws; wins vs defeats; and draws vs defeats for squad average}

As we stated before, to the best of our knowledge, this was the first study to report external and internal TL data from the entire season organized by match result (win, draw and defeat). Another major point is that the team selected for analysis was an elite European soccer team and this therefore means a larger amount of data from weeks that preceded wins $(78.43 \%)$ compared to data that preceded draws $(5.88 \%)$ or defeats $(15.69 \%)$.

Regarding internal TL, for MD-5, scores from fatigue and DOMS were higher in draws than wins or defeats, which may be associated with higher physical demand preceding this match outcome. Total HI score was higher because fatigue and DOMS scores were also higher. Duration of training sessions and s-RPE revealed differences for all match scores, but lower for draws, which may be related to the higher HI scores previously reported. External TL variables did not present any differences.

Furthermore, s-RPE seemed to have a relevant role in MD-5 and MD-2 by dictating the winning of matches, however some intensity is required to be applied in training sessions because s-RPE values were higher when there was a defeat and lower when there was a draw. However, Barrett et al. [30] did not find substantial effects for s-RPE related to match result. Thus, future studies are required to interpret our data.

Regarding MD-1, our results corroborated some studies where it was found that DOMS, fatigue, stress and sleep quality remain similar for win, draw and defeat [18,31].

On the other hand, internal TL, HI, had an important role in MD-5 and MD+1. For MD+1, scores from HI were higher than all MD- $(5,4,3,2,1)$ and there were significant differences between win vs draw but none for win vs defeat. For instance, Fessi and Moalla [32] stated that DOMS was similar between match results and stress, fatigue and bad sleep quality were higher after a lost match in Qatar league. Furthermore, Fessi and Moalla [32] stated showed that regarding the match result, players rated and average score of 3.5 au for both perceived sleep quality and stress, 4 au for fatigue and 5.5 au for DOMS in the following day of the match. These results did not corroborate ours (see Table 1). Once again, it is important to reinforce that Fessi and Moalla study [32] came from Qatar league. However, it was not provided by our study or Fessi and Moalla [32] what were the aim of the team (e.g. if they are playing to win the league or for another place). Therefore, further investigation is needed to support these findings. On the other hand, our results are in line with Fessi and Moalla [32] for stress in the 
following day to a draw as opposed to win or defeat but in opposition to Abbott et al. [31] who stated that stress increased, and sleep quality decreased in the following day after a defeat in the under 23 Premier league 2 division. Therefore, it should be considered that both results (draw and defeat) are negative and could have some negative impact in soccer players as observed in rugby players [33] and female soccer players [34].

In opposition to our data, Conde et al. [19] found no difference on recovery measured 40 h 40 hours after the matches by perceived recovery scale. Although our study did not use the same scale, we speculate that we could obtain different results in MD+1.

In addition, HI scores revealed few variations in days prior to the match with the highest values being reported in the day after the match supporting the statement that matches were the most demanding workload of each week [ 4,18,32]. It should be noticed that HI is very subjective to each player and average data should be carefully interpreted and the summation of the four categories that generates total HI should be interpreted carefully, once fatigue, stress and DOMS used 1-7 scale in which 1 is very, very low and 7 is very, very high, but sleep quality used 1-7 scale in which 1 is very, very good and 7 is very, very bad [9]. The results could be different if we had an inversed scale related to the sleep quality or the other items of HI.

In $\mathrm{MD}+1$, there were no significant differences for s-RPE which is line with Abbott et al. [31] study.

Regarding external TL, it was found that HSRD was important from MD-3 to MD-1. The results of HSRD are in line with the s-RPE by revealing that to win matches, it is necessary to apply some intensity, but not the highest that could lead to a non-positive result. These findings were in line with some studies that reported a need to apply HSRD to win matches [35,36]. HSRD is associated with the decisive short and intense actions that characterise the effort made by elite soccer players [37,38]. Further, HSRD seems to be a better sensitive indicator of performance than total distance covered [39].

Total distance covered was lower in MD-3 and MD-2, but higher in MD-1 in order to win matches. It seems to be relevant to take these notes in consideration to have players at their best and avoiding accumulated fatigue during the week. It is not clear why is this observed because and to the best of our knowledge, according with Lago et al. [40] and Moalla et al._:[41]. players cover greater total distance for matches won compared to matches drawn or lost however these authors did not analyse training data, only match data. On the other hand, Andrzejewski et al. [42] observed similar data between the different match results. In addition, and according to our data AvS should be higher in MD-1 in order to win matches. Once again, future studies are needed to find what could possibly be the best training periodization approaches to win matches.

In $\mathrm{MD}+1$, there were more minutes during training sessions in win $>$ draw $>$ defeat and there were no significant differences for external TL variables. However, this study points to similar results to the study of Owen et al, [17]. that also found lower average speeds after a win compared to a draw or defeat, although Brito et al. [18] found higher weekly TLs preceding a defeat or draw when compared to a win. It is important to refer that Owen et al. [17] study analysed an Elite European soccer team during a mesocycle of 6 weeks and the Brito et al. [18] study analysed under-19 players from a first league club in France during the entire in-season.

Gabbett [43] suggested that physical effort is higher for match score win vs defeat. Despite the fact this statement is regarding the match and not training session, we could speculate that weeks preceding wins had higher TL than weeks preceding draw or defeat, but that was not observed in our data because only AvS was higher in weeks preceding wins, and the other internal and external TL were higher in weeks preceding draws or defeats. Furthermore, training sessions from different match day minus were not characterized over the in-season, which 
means that it is not possible to provide more insight regarding what kind of exercises were made. Exercises, such as small-sided games, position games (movements with/without ball but without the dynamic of a real game), simulations of 11 vs. 11 game, fitness training, large-sided games, technical training, had different physiological impacts on aerobic or anaerobic.

The reduced variance of in-seasonal TL in the MD- between different match results is supported by other study which has suggested that the main emphasis of exercise training sessions was on the maintenance of the physical fitness to accommodate competitive seasonal match demands [17]. On the other hand, the tapered model used in this study only revealed decreases when match-day is close, being MD-1 the day with lower internal and external TL values [16,17]. One of the justifications highlighted by Malone et al. [16] is that coaches usually reduce the TL the day preceding the match to increase player readiness. In addition, given the limited time for recovery between competitive matches, coaches commonly concentrate higher training intensity sessions in the middle of the week cycle in order to prevent excessive loading on the immediate days [18]. Other authors have also reported that higher TL appeared in the beginning to middle days of the weekly-microcycle to promote stimulus and contribute to a better development of the players [16,44]. Additionally, there is a mismatch between the training load intended by the coaches and its perception from the players that needs to be considered [45].

Additionally, the precision of GPS data is greatest during lower to moderate speed activities completed, over longer distances. Despite this, the actual data rate acquisition have effectiveness and can be evaluated by its measurement properties (i.e., reliability, validity, sensitivity), so the higher-intensity activities present a greater perceived practical importance, as they occur as part of key match or training activities. They can be used as indicators of physical performance and they are theoretically associated with positive and negative outcomes, such as fitness or injury. Although the speed thresholds defined in the present study used the absolute method and do not allow the comparison of physical performance between players and positional roles but allow between match day minus of different microcycles. The training load measured after games with different results does not allow a consensual rationale and makes it difficult to speculate on trends in the direction of loads applied depending on the result. However, it appears that other factors may eventually influence the rationality of the applied load, namely the preparation of the next game and the modification of the load with a more anaerobic or aerobic nature and longer or shorter duration of training sessions and total distances covered.

\subsection{Practical applications and limitations}

This study provides useful information relating to the TL employed by an elite soccer team that played in a European Competition. It provides further evidence of the value of using the combination of different measures of TL to fully evaluate the patterns observed across in-season. For coaches and practitioners, the current study generates reference values for elite soccer players' training design.

However, it is important to remember that the in-season match-day-minus training comparison was analysed by mean values. In addition, the in-season period has around 40 weeks and during each week (microcycle of a 7day period) different patterns occur, as mentioned before. For example, there are weeks with 6, 5, 4, 3 and 2 training sessions due to the competitive schedule.

Furthermore, and as reported in our previous studies [3,4], the small sample size does not allow to generalize the results even with players from a top-class European soccer team. Moreover, this study included one top elite European soccer team that played European matches and for that reason it may not be representative of the customary training demands of other domestic teams. Then, the horizontal dilution of precision is not given by 
the GPS technology used what should be considered as a limitation as stated by Beato et al. [20]. Finally, accelerations or decelerations were not used in this study what may provide some new findings.

Therefore, more research is needed with larger numbers regarding soccer players, teams, and/or soccer matches analysed. It is important to highlight that there are several other variables that could have affected variables analysed in this study such as tactical and technical performance and other situational factor such as match location and quality of the opponents that were not controlled for this study.

\section{Conclusions}

Although the present findings may reflect only the particular top European soccer team that participated in UEFA Champions League, we may conclude that internal and external TL showed some significantly different values when comparing match results, which could mean that TL can influence the match result when preceding a win, draw, or defeat.

Despite its limitations, this work provides novel findings regarding the contextual variables of match result from a top European soccer team and appears to be relevant as a first recommendation for coaches and for future research. More expansive investigations should be conducted, especially in teams that do not play European competitions or teams that play outside of Europe. Also, they should find reference intervals for TL in order to win matches for weekly schedules, with different number of matches per week.

Finally, from a practical perspective, coaches should consider the information presented in this paper to adjust their own training sessions and to apply a proper load in order to win soccer matches. In addition, this study highlights the use of HI scores specially the day following the match and external TL data every training session when match result variable is considered.

\section{Funding}

This project was supported by the Portuguese Foundation for Science and Technology, I.P., Grant/Award Numbers UIDP/04748/2020, UIDB/04045/2020 and UIDP/04045/2020.

\section{Declarations of Competing Interest}

None.

\section{Acknowledgements}

The authors would like to thank the team's coaches and players for their cooperation during all data collection procedures.

\section{References}

The corrections made in this section will be reviewed and approved by a journal production editor. The newly added/removed references and its citations will be reordered and rearranged by the production team.

[1] Anderson L., Orme P., Di Michele R., Close G.L., Morgans R., Drust B., Morton J.P., Quantification of training load during one-, two- and three-game week schedules in professional 
soccer players from the English Premier League: implications for carbohydrate periodisation, J. Sports Sci. 34 (13) (2016) 1250-1259, doi:10.1080/02640414.2015.1106574.

[2] Nédélec M., McCall A., Carling C., Legall F., Berthoin S., Dupont G., Recovery in soccer: part I - post-match fatigue and time course of recovery, Sports Med. 42 (2012) 997-1015.

[3] Oliveira R., Brito J.P., Martins A., Mendes B., Calvete F., Carriço S., Ferraz R., Marques M.C., In-season training load quantification of one-, two- and three-game week schedules in a top European professional soccer team, Physiol. Behav. 201 (2019) 146-156 a, doi:10.1016/j.physbeh.2018.11.036.

[4] Oliveira R., Brito J.P., Martins A., Mendes B., Marinho D., Ferraz R., Marques M.C., In-season internal and external training load quantification of an elite European soccer team, PLoS ONE 14 (4) (2019 b). 10.1371/journal e0209393 pone.0209393.

[5] Morgans R., Adams D., Mullen R., McLellan C., Williams M., M. Technical and physical performance over and English championship league season, Int. J. Sport Sci. Coach. 9 (5) (2014) 493-503.

[6] Taylor K., Fatigue monitoring in high performance sport: a survey of current trends, J. Aus. Strength Cond. 20 (2012) 12-23.

[7] Wallace L.K., Slattery K.M., Coutts A.J., A comparison of methods for quantifying training load: relationships between modelled and actual training responses, Eur. J. Appl. Physiol. 114 (1) (2014) 11-20, doi:10.1007/s00421-013-2745-1.

[8] Impellizzeri F.M., Rampinini E., Marcora S.M., Physiological assessment of aerobic training in soccer, J. Sports Sci. 23 (2005) 583-592, doi:10.1080/02640410400021278.

[9] Hooper S.L., Mackinnon L.T., Monitoring overtraining in athletes, Sports Med. 20 (5) (1995) 321-327 Retrieved from http://link.springer.com/10.2165/ 00007256-199520050-00003.

[10] Foster C., Monitoring training in athletes with reference to overtraining syndrome, Med. Sci. Sports Exerc. 30 (1998) 1164-1168, doi:10.1097/00005768-199807000-00023.

[11] Haddad M., Chaouachi A., Wong D.P., Castagna C., Hambli M., Hue O., Chamari K., Influence of fatigue, stress, muscle soreness and sleep on perceived exertion during submaximal effort, Physiol. Behav. 119 (2013) 185-189, doi:10.1016/j.physbeh.2013.06.016.

[12] Clemente F., Mendes B., Nikolaidis P., Calvete F., Carriço S., Owen A., Internal training load and its longitudinal relationship with seasonal player wellness in elite professional soccer, Physiol. Behav. 179 (2017) 262-267, doi:10.1016/j.physbeh.2017.06.021.

[13] Owen A.L., Wong P., Dunlop G., Groussard C., Kebsi W., Dellal A., Morgans R., Zouhal H., High intensity training and salivary immunoglobulin - a responses in professional top-level soccer players: effect of training intensity, J. Strength Cond. Res. 30 (9) (2016) 2460-2469, doi:10.1519/JSC.0000000000000380.

[14] Gaudino P., Iaia F.M., Alberti G., Strudwick A.J., Atkinson G., Gregson W., Monitoring training in elite soccer players: a systematic bias between running speed and metabolic power data, Int. J. 
Sports Med. 34 (11) (2013) 963-968, doi:10.1055/s-0033-1337943.

[15] Impellizzeri F.M., Rampinini E., Coutts A.J., Sassi A., Marcora S.M., Use of RPE-based training load in soccer, Med. Sci. Sports Exerc. 36 (6) (2004) 1042-1047.

[16] Malone J., Di Michele R., Morgans R., Burgess D., Morton J., Drust B., Seasonal training-load quantification in Elite English Premier League soccer players, Int. J. Sports Physiol. Perform. 10 (2015) 489-497, doi:10.1123/ijspp.2014-0352.

[17] Owen A.L., Lago-Peñas C., Gómez M.A., Mendes B., Dellal A., Analysis of a training mesocycle and positional quantification in elite European soccer players, Int. J. Sport Sci. Coach. 12 (5) (2017) 665-676, doi:10.1177/1747954117727851.

[18] Brito J., Hertzog M., Nassis G.P., Do match-related contextual variables influence training load in highly trained soccer players?, J. Strength Cond. Res. 2 (2016) 393-399.

[19] Conde J., Alves D., Novack L., Carneiro C., Cruz R., Osiecki R., Comparisons of recovery, external and internal load by playing position and match outcome in professional soccer, Motriz 24 (1) (2018) e1018140, doi:10.1590/s1980-657420180001000310.1590/s19806574201800010003.

[20] Beato M., Devereux G., Stiff A., Validity and reliability of global positioning system units (STATSports Viper) for measuring distance and peak speed in sports, J. Strength Cond. Res. 32 (10) (2018) 2831-2837, doi:10.1519/jsc.0000000000002778.

[21] Maddison R., Ni Mhurchu C., Global positioning system: a new opportunity in physical activity measurement, Int. J. Behav. Nutr Phys Act. 6 (2009) 73.

[22] Buchheit M., Monitoring training status with hr measures: do all roads lead to Rome?, Front. Physiol. 5 (2014) 73, doi:10.3389/fphys.2014.00073.

[23] Borg G., Perceived exertion as an indicator of somatic stress, Scand. J. Rehabil. Med. 2 (1970) 92-98.

[24] Foster C., Florhaug J.A., Franklin J., Gottschall L., Hrovatin L.A., Parker S., Doleshal P., Dodge C., A new approach to monitoring exercise training, J. Strength Cond. Res. 15 (2001) 109-115.

[25] Foster C., Hector L., Welsh R., Schrager M., Green M., Snyder A., Effects of specific versus cross-training on running performance, Eur. J. Appl. Physiol. Occup. Physiol. 70 (4) (1995) 367372, doi:10.1007/BF00865035.

[26] Hopkins W.G., Spreadsheet for analysis of controlled trials with adjustment for a subject characteristics, J. Sport. Sci. 10 (2006) 46-50.

[27] Renfree A., Martin L., Micklewright D., Gibson A., Application of decision-making theory to the regulation of muscular work rate during self-paced competitive endurance activity, Sports Med. 44 (2) (2014) 147-158, doi:10.1007/s40279-013-0107-0.

[28] Ferraz R., Gonçalves B., Van Den Tillaar R., Jimenez S., Sampaio J., Marques M., Effects of knowing the task duration on players' pacing patterns during soccer small-sided games, J. Sport 
Sci. (2017) 1-7, doi:10.1080/24733938.2017.1283433.

[29] Ferraz R., Gonçalves B., Coutinho D., Marinho D., Sampaio J., Marques M., Pacing behaviourof players in team sports: influence of match status manipulation and task durationknowledge, PLoS ONE 13 (2) (2018) e0192399, doi:10.1371/journal.pone.0192399.

[30] Barrett S., McLaren S., Spears I., Ward P., Weston M., The influence of playing position and contextual factors on soccer players' match differential ratings of perceived exertion: a preliminary investigation, Sports 6 (1) (2018) 13, doi:10.3390/sports601001.3.

[31] Abbott W., Brownlee T.E., Harper L.D., Naughton R.J., Clifford T., The independent effects of match location, match result and the quality of opposition on subjective wellbeing in under 23 soccer players: a case study, Res. Sports Med. 26 (3) (2018) 262-275, doi:10.1080/15438627.2018.1447476.

[32] Fessi M.D., Moalla W., Postmatch perceived exertion, feeling, and wellness in professional soccer players, Int. J. Sports Physiol. Perform. 13 (2018) 631-637, doi:10.1123/ijspp.2017-0725.

[33] Polman R., Nicholls A.R., Cohen J., Borkoles E., The influence of game location and outcome on behaviour and mood states among professional rugby league players, J. Sports Sci. 25 (13) (2007) 1491-1500.

[34] Oliveira T., Gouveia M.J., Oliveira R.F., Testosterone responsiveness to winning and losing experiences in female soccer players, Psychoneuroendocrinology 34 (7) (2009) 1056-1064.

[35] Dellal A., Chamari K., Wong D.P., Ahmaidi S., Keller D., Barros R., Bisciotti G.N., Comparison of physical and technical performance in European soccer match-play, Eur. J. Sport Sci. 11 (2011) $51-59$.

[36] Chmura P., Andrzejewski M., Konefal M., Mroczek D., Rokita A., Chmura J., Analysis of motor activities of professional soccer players during the 2014 World Cup in Brazil, J. Hum. Kinet. 56 (2017) 187-195.

[37] Stolen T., Chamari K., Castagna C., Physiology of soccer: an update, Sports Med. 35 (2005) 501536.

[38] Djaoui L., Wong D.P., Pialoux V., Hautier C., Da Silva C.D., Chamari K., Dellal A., Physical activity during a prolonged congested period in a top-class European football team, Asian J. Sports Med. 5 (2014) 47-53.

[39] Bradley P.S., Carling C., Archer D., Roberts J., Dodds A., Di Mascio M., Paul D., Diaz A.G., Peart D., Krustrup P., The effect of playing formation on high-intensity running and technical profiles in English FA Premier League soccer matches, J. Sports Sci. 29 (2011) 821-830.

[40] Lago C., Casais L., Dominguez E., Sampaio J., The effects of situational variables on distance covered at various speeds in elite soccer, Eur. J. Sport Sci. 10 (2010) 103-109.

[41] Moalla W., Fessi M.S., Makni E., Dellal A., Filetti C., Di Salvo V., Chamari K., Association of physical and technical activities with partial match status in a soccer professional team, J. Strength Cond. Res. (2017) 1-22. 
[42] Andrzejewski M., Konefał M., Chmura P., Kowalczuk E., Chmura J., Match outcome and distances covered at various speeds in match play by elite German soccer players, Int. J. Perform. Anal. Sport. 16 (3) (2017) 817-828.

[43] Gabbett T.J., Influence of the opposing team on the physical demands of elite rugby league match play, J. Strength Cond. Res. 27 (6) (2013) 1629-1635, doi:10.1519/JSC.0b013e318274f30e.

[44] Owen A.L., Dunlop G., Rouissi M., Haddad M., Mendes B., Chamari K., Analysis of positional training loads (ratings of perceived exertion) during various-sided games in European professional soccer players, Int. J. Sports Sci. Coach. 11 (3) (2016) 374-381 Available from http://journals.sage pub.com/doi/10.1177/1747954116644064.

[45] Brink M.S., Frencken W.G., Jordet G., Lemmink K.A., Coaches' and players’ perceptions of training dose: not a perfect match, Int. J. Sports Physiol. Perform. 9 (2014) 497-502.

\section{Highlights}

- Total distance and average speed were higher in training that preceded win.

- High-speed running distance was similar in training that preceded win and defeat.

- Hooper index was lower after a win than a draw or defeat.

- S-RPE seems to vary through the week with no relation to the match result.

\section{Queries and Answers}

Query: Please confirm that givennames and surnames have been identified correctly.

Answer: Yes

Query: Please check table 1 for correctness.

Answer: We put the MD-5, MD-4, MD-3, MD-2, MD-1 and MD+1 in bold.

Query: Please check reference for article title and correct if required.

Answer: The reference is correct.

Query: Please check reference for journal title and correct if required.

Answer: Now the reference is correct.

Query: Volume is not present. Please check.

Answer: The reference is correct. 
